\title{
ASSOCIATION OF CANADIAN UNIVERSITY DEPARTMENTS OF ANESTHESIA
}

POSTGRADUATE FELLOWSHIPS IN ANESTHESIA

\begin{tabular}{|c|c|c|c|}
\hline $\begin{array}{l}\text { Dalhousie University } \\
\text { Halifax, Nova Scotia }\end{array}$ & $\begin{array}{l}\text { 1 - Chronic Pain } \\
\text { Prerequisite: Eligibility for full } \\
\text { licence in Nova Scotia }\end{array}$ & $\begin{array}{l}\text { July } 12009 \\
\text { - June } 302010\end{array}$ & $\begin{array}{l}\text { Dr. Ian Beauprie, Education Director, Pain Management Unit, QEII Health } \\
\text { Sciences Centre, Halifax, Nova Scotia B3H 2A7. Phone: 902-473-6428; } \\
\text { Fax: 902-473-4126; E-mail: i.beauprie@dal.ca }\end{array}$ \\
\hline $\begin{array}{l}\text { McGill University } \\
\text { Montreal, Quebec }\end{array}$ & $\begin{array}{l}\text { 1- Obstetric } \\
\text { 1 - Pain Management } \\
\text { 1 - General Pediatric } \\
\text { 1 - Pediatric Pain } \\
\text { 1 - Clinical Reseach } \\
\text { 1 - Regional Anesthesia } \\
\text { 1 - Cardiac Anesthesia }\end{array}$ & $2008 / 2009$ & $\begin{array}{l}\text { Dr. Gilles Plourde, Director, Fellowship Program, Department of Anesthesia, } \\
\text { McGill University, Royal Victoria Hospital, 687 Pine Avenue West, Room } \\
\text { F9.16, Montreal, Quebec H3A 1A1. Phone: 514-934-1934, ext. 36546; Fax: } \\
\text { 514-843-1488; E-mail: sandra.cardoso@muhc.mcgill.ca. } \\
\text { Note: The dates are constantly changing and interested candidates should } \\
\text { contact us for their availability. }\end{array}$ \\
\hline
\end{tabular}

\begin{tabular}{|c|c|c|c|}
\hline $\begin{array}{l}\text { University of Toronto } \\
\text { Toronto, Ontario }\end{array}$ & & & \\
\hline $\begin{array}{l}\text { Toronto General } \\
\text { Hospital }\end{array}$ & $\begin{array}{l}2 \text { - Thoracic (Clinical/ } \\
\text { Research) } \\
8 \text { - Cardiac \& Intensive Care } \\
\text { (ICU/Clinical/Research/ } \\
\text { TEE) } \\
4 \text { - Transplantation (Heart/ } \\
\text { Lung/Liver) } \\
\text { 1 - Airway }\end{array}$ & $2008 / 2009$ & $\begin{array}{l}\text { Ms. Julie Nigro, Department of Anesthesia and Pain Management, University } \\
\text { Health Network, Toronto General Hospital, 200 Elizabeth Street, EN3-438, } \\
\text { Toronto, Ontario M5G 2C4. Phone: 416-340-3567; Fax: 416-340-3696; } \\
\text { E-mail: julie.nigro@uhn.on.ca }\end{array}$ \\
\hline $\begin{array}{l}\text { Toronto Western } \\
\text { Hospital }\end{array}$ & $\begin{array}{l}\text { 1 - Pain (Acute/Chronic/ } \\
\text { Clinical Research) } \\
4 \text { - Regional Anesthesia } \\
\text { (Clinical/Research) } \\
3 \text { - Neurosurgical } \\
\text { (Clinical/Research) } \\
3 \text { - Ambulatory Anesthesia } \\
\text { (Clinical/Research) }\end{array}$ & $2008 / 2009$ & $\begin{array}{l}\text { Dr. Atul Prabhu, Department of Anesthesia and Pain Management, University } \\
\text { Health Network, Toronto Western Hospital, } 399 \text { Bathurst Street, } \\
\text { McL } 2 \text { - 405, Toronto Ontario M5T 2S8. Phone: 416-603-5118; } \\
\text { Fax: 416-603-6494; E-mail: atul.prabhu@uhn.on.ca }\end{array}$ \\
\hline Mount Sinai Hospital & $\begin{array}{l}6 \text { - Obstetrical (Clinical/ } \\
\text { Research } \\
6 \text { - Clinical Fellowships }\end{array}$ & $2008 / 2009$ & $\begin{array}{l}\text { Dr. Gordon Fox, Department of Anesthesia, Mount Sinai Hospital, } 600 \\
\text { University Avenue, 1514, Toronto Ontario M5G 1X5. Phone: 416-586-4800 } \\
\text { ext. 2698; Fax: 416-586-8664; E-mail: gfox@mtsinai.on.ca }\end{array}$ \\
\hline $\begin{array}{l}\text { Sunnybrook and } \\
\text { Women's College } \\
\text { Health Sciences Centre }\end{array}$ & $\begin{array}{l}\text { 1 - Chronic Pain - must have } \\
\text { previous experience (Clinical/ } \\
\text { Research) } \\
\text { 1 - Cardiac Anesthesia } \\
\text { (Clinical/Research) } \\
4 \text { - Regional Anesthesia } \\
\text { (Clinical/Research) } \\
\text { 1 - Simulation/Research }\end{array}$ & $2008 / 2009$ & $\begin{array}{l}\text { Dr. Gil Faclier, Anesthetist-in-Chief, Dr Martha ODriscoll, Director } \\
\text { Fellowship Program, Department of Anesthesia, Sunnybrook Health Sciences } \\
\text { Centre, } 2075 \text { Bayview Avenue, M3-200, Toronto, Ontario M4N 3M5. } \\
\text { Phone: 416-480-4864; Fax: 416-480-6039; E-mail: gil.faclier@sw.ca }\end{array}$ \\
\hline
\end{tabular}

\begin{tabular}{|c|c|c|c|}
\hline & $\begin{array}{l}2 \text { - Obstetrical Clinical/ } \\
\text { Research }\end{array}$ & $2008 / 2009$ & $\begin{array}{l}\text { Dr. Jean Kronberg, Site Chief, Department of Anesthesia, Women’s College } \\
\text { Campus, Sunnybrook and Women’s College Health Sciences Centre, } \\
76 \text { Grenville Street, Toronto, Ontario M5S 1B2. Phone: 416-323-6269; } \\
\text { E-mail: jean.kronberg@sw.ca } \\
\text { Dr. Stephen Halpern, Director, Obstetrical Anesthesia, Department of } \\
\text { Anesthesia, Women's College Campus, Sunnybrook and Women's College } \\
\text { Health Sciences Centre, 76 Grenville Street, Toronto, Ontario M5S 1B2. } \\
\text { Phone: 416-323-6269; E-mail: Stephen.halpern@sw.ca } \\
\text { Dr. Pamela Angle, Associate Director, Research, Obstetrical Anesthesia, } \\
\text { Department of Anesthesia, Women's College Campus, Sunnybrook and } \\
\text { Women's College Health Sciences Centre, 76 Grenville Street, Toronto, } \\
\text { Ontario M5S 1B2. Phone: 416-323-6269; E-mail: pamela.angle@sw.ca }\end{array}$ \\
\hline $\begin{array}{l}\text { Hospital for Sick } \\
\text { Children }\end{array}$ & $\begin{array}{l}10 \text { - Pediatric Anesthesia } \\
\text { Clinical/Research } \\
\text { 1 - Pediatric Chronic Pain } \\
\text { (Clinical/Research) } \\
\text { 1 - Research } \\
\text { 1 - Pediatric Anesthesia } \\
\text { \& Pediatric ICU (2-year } \\
\text { program - Clinical) }\end{array}$ & $2008 / 2009$ & $\begin{array}{l}\text { Dr. L. Roy, Anesthetist-in-Chief, Department of Anesthesia, Hospital for } \\
\text { Sick Children, } 555 \text { University Avenue, Toronto, Ontario M5G IX8. } \\
\text { Phone: 416-813-7445; Fax: 416-813-7543; E-mail: lawrence.roy@sickkids.ca. } \\
\text { Information available at: http://www.sickkids.ca/anaesthesia }\end{array}$ \\
\hline
\end{tabular}




\section{ASSOCIATION OF CANADIAN UNIVERSITY DEPARTMENTS OF ANESTHESIA}

POSTGRADUATE FELLOWSHIPS IN ANESTHESIA

\begin{tabular}{|c|c|c|c|}
\hline & $\begin{array}{l}10 \text { - Pediatric Critical Care } \\
\text { (Clinical) } \\
2 \text { - Pediatric Critical Care } \\
\text { (Research) }\end{array}$ & $2008 / 2009$ & $\begin{array}{l}\text { Dr. Peter Cox, Education Program Director, Department of Critical Care } \\
\text { Medicine, Hospital for Sick Children, } 555 \text { University Avenue, Toronto, } \\
\text { Ontario M5G 1X8. Phone: 416-813-6484; Fax: 416-813-5093; } \\
\text { Dept. fax: 416-813-7299. }\end{array}$ \\
\hline St. Michael's Hospital & $\begin{array}{l}\text { 4- Cardiac Anesthesia/Echo/ } \\
\text { CV-ICU (Clinical/Research) } \\
2 \text { - Neurotrauma/Neuro-ICU } \\
\text { (Clinical/Research) } \\
1 \text { - Research in Education/ } \\
\text { Simulation (Clinical) } \\
\text { 1 - Acute \& Chronic Pain } \\
\text { 1- Airway/Simulation } \\
\text { (Clinical) } \\
\text { 1- Regional Anesthesia and } \\
\text { Ultrasound Guided Techniques }\end{array}$ & January 2009 & $\begin{array}{l}\text { Dr. P.L. Houston, Anesthetist-in-Chief, Department of Anesthesia, St. } \\
\text { Michael's Hospital, } 30 \text { Bond Street, Toronto, Ontario M5B 1W8. Phone: } \\
\text { 416-864-5071; Fax: 416-864-6014; E-mail: houstonp@smh.toronto.on.ca } \\
\text { More detailed information on these fellowship positions may be obtained from } \\
\text { the Department of Anesthesia home page at: } \\
\text { http://www.anesthesia.utoronto.ca }\end{array}$ \\
\hline \multirow[t]{3}{*}{$\begin{array}{l}\text { University of Manitoba } \\
\text { Winnipeg, Manitoba }\end{array}$} & $\begin{array}{l}\text { 1-2 Acute Pain, Chronic Pain } \\
\text { and Regional Anesthesia }\end{array}$ & July 1,2009 & $\begin{array}{l}\text { Dr. Ian Sutton, Pain Fellowship Director, Pain Clinic, MS261, } 820 \text { Sherbrook } \\
\text { St. Winnipeg, Manitoba R3A IR9. }\end{array}$ \\
\hline & 1- Pediatric Anesthesia & July 1,2009 & $\begin{array}{l}\text { Dr. Heather Brownell, Pediatric Fellowship Director, Department of } \\
\text { Anesthesia, LB315, } 60 \text { Pearl St. Winnipeg, Manitoba R3E 1X2. }\end{array}$ \\
\hline & 2 Cardiac Anesthesia/TEE & July 2008 & $\begin{array}{l}\text { Dr. Scott Mackenzie, Director, Cardiac Anesthesia, WRHA Cardiac Sciences } \\
\text { Program, St. Boniface General Hospital, Room Z3045, } 409 \text { Tache Avenue, } \\
\text { Winnipeg, Manitoba R2H 2A6. Phone: 204-237-2381. }\end{array}$ \\
\hline \multirow[t]{3}{*}{$\begin{array}{l}\text { University of Ottawa } \\
\text { Ottawa, Ontario }\end{array}$} & $\begin{array}{l}\text { 1- Obstetrical Anesthesia } \\
1 \text { - Thoracic Anesthesia } \\
\text { 2- Regional Anesthesia } \\
1 \text { - Vascular Anesthesia } \\
\text { 1 - Perioperative Medicine } \\
\text { 1 - Simulator } \\
\text { 1 - Chronic Pain }\end{array}$ & July 2009 & $\begin{array}{l}\text { Dr. Homer Yang, Chief, Department of Anesthesia, The Ottawa Hospital, } \\
\text { Civic Campus, 1053 Carling Avenue, Room B309, Ottawa, Ontario K1Y 4E9. } \\
\text { Phone: 613-761-4940; Fax: 613-761-5032. }\end{array}$ \\
\hline & 2 - Cardiac Anesthesia & July 2009 & $\begin{array}{l}\text { Dr. Jean-Yves Dupuis, Fellowship Coordinator, University of Ottawa } \\
\text { Heart Institute, } 40 \text { Ruskin Street, Room H213, Ottawa, Ontario } \\
\text { K1Y 4W7. Phone: 613-761-4379; Fax: 613-761-4925; } \\
\text { E-mail: jydupuis@ottawaheart.ca }\end{array}$ \\
\hline & 2 - Pediatric Anesthesia & July 2009 & $\begin{array}{l}\text { Dr. Gary Johnson, Chief, Department of Anesthesia, Children's Hospital of } \\
\text { Eastern Ontario, 401 Smyth Road, Room 3348, Ottawa, Ontario K1H 8L1. } \\
\text { Phone: 613-737-2431; Fax: 613-738-4815. }\end{array}$ \\
\hline $\begin{array}{l}\text { University of Calgary } \\
\text { Calgary, Alberta }\end{array}$ & 1-Pediatric Anesthesia & July $1^{\text {st }} 2009$ & $\begin{array}{l}\text { Dr. Debbie McAllister, Department of Anesthesia, Alberta Children's Hospital, } \\
2888 \text { Shaganappi Trail, N.W. Calgary, Alberta T3B 6A8. Phone: 403-944- } \\
7810 \text {; Fax: 403-944-7606. }\end{array}$ \\
\hline \multirow[t]{2}{*}{$\begin{array}{l}\text { McMaster University } \\
\text { Hamilton, Ontario }\end{array}$} & $\begin{array}{l}\text { Hamilton Health Sciences } \\
\text { Corporation } \\
2 \text { - Pain } \\
2 \text { - Cardiac Anesthesia } \\
\text { 1 - Pediatric }\end{array}$ & $2009 / 2010$ & $\begin{array}{l}\text { Dr. N. Buckley, Chair, Department of Anesthesia, McMaster University, Room } \\
2 \text { U1, } 1200 \text { Main Street West, Hamilton, Ontario L8N 3Z5. Phone: 905-521- } \\
2100 \text {, ext. 75166; Fax: 905-523-1224. }\end{array}$ \\
\hline & $\begin{array}{l}\text { Hamilton Health Sciences } \\
\text { St. Joseph's Health Care } \\
4 \text { - Critical Care Residency }\end{array}$ & $2009 / 2010$ & $\begin{array}{l}\text { Director, Critical Care Medicine Residency Program c/o Department of } \\
\text { Anaesthesia, McMaster University, Room 2U, 1200 Main Street West, } \\
\text { Hamilton, Ontario L8N 3Z5. Phone: 905-521-2100, ext. 75180, } \\
\text { Fax: 905-523-1224. }\end{array}$ \\
\hline $\begin{array}{l}\text { The University of } \\
\text { Western Ontario } \\
\text { London, Ontario }\end{array}$ & $\begin{array}{l}\text { Up to 17- Clinical/Research } \\
\text { Cardiac and CSRU, } \\
\text { Neuro, Transplant, Pain } \\
\text { Management, Vascular- } \\
\text { Thoracic, Obstetrical, } \\
\text { Regional Anesthesia, Medical } \\
\text { informatics; ambulatory } \\
\text { individually or in combination } \\
\text { 1 - Research (W.E. Spoerel } \\
\text { Research Fellowship) }\end{array}$ & July 12009 & $\begin{array}{l}\text { Dr. Daniel T. Bainbridge, Director, Fellowship Program, } \\
\text { Department of Anesthesia \& Perioperative Medicine, } \\
\text { London Health Sciences Centre, University Campus, } 339 \text { Windermere Road, } \\
\text { London, Ontario N6A 5A5. Phone: 519-663-3384; Fax: 519-663-2957; } \\
\text { E-mail: daniel.bainbridge@lhsc.on.ca } \\
\text { Website: www.uwoanesthesia.ca }\end{array}$ \\
\hline
\end{tabular}




\section{ASSOCIATION OF CANADIAN UNIVERSITY DEPARTMENTS OF ANESTHESIA}

POSTGRADUATE FELLOWSHIPS IN ANESTHESIA

\begin{tabular}{|c|c|c|c|}
\hline & $\begin{array}{l}\text { Critical Care } \\
2 \text { - Ministry of Health } \\
\text { (Ontario) }\end{array}$ & 2009 & $\begin{array}{l}\text { Dr. David Leasa, Postgraduate Medical Education Director, Program in } \\
\text { Critical Care, London Health Sciences Centre, University Campus, } \\
339 \text { Windermere Road, London, Ontario N6A 5A5. Phone: 519-663-3291; } \\
\text { Fax: 519-663:8813; E-mail:david.leasa@lhsc.on.ca } \\
\text { Website: www.lhsc.on.ca/programs/critcare/pge }\end{array}$ \\
\hline \multirow[t]{4}{*}{$\begin{array}{l}\text { University of British } \\
\text { Columbia } \\
\text { Vancouver, British } \\
\text { Columbia }\end{array}$} & $\begin{array}{l}\text { BC Children's Hospital } \\
2 \text { - Clinical/Research } \\
\text { (Pediatric anesthesia only) }\end{array}$ & 2009-2010 & $\begin{array}{l}\text { Dr. Carolyne J. Montgomery, Fellowship Director, BC Children’s Hospital, } \\
\text { Room 1L7 - } 4480 \text { Oak Street Vancouver, BC V6H 3V4. } \\
\text { Phone: 604-875-2711; Fax: 604-875-3221; E-mail: cmontgomery@cw.bc.ca }\end{array}$ \\
\hline & $\begin{array}{l}\text { BC Children's Hospital } \\
\text { 1 - Pediatric Pain Fellowship }\end{array}$ & 2008-2010 & $\begin{array}{l}\text { Dr. Gillian Lauder, Director of the Integrated Pain Service, } \\
\text { BC Children's Hospital, E400 Shaughnessy Bldg. } \\
4480 \text { Oak Street Vancouver BC V6H 3V4. Phone: 604-875-2345, ext. 5955; } \\
\text { Fax: 604-875-2767; E-mail: glauder@cw.bc.ca }\end{array}$ \\
\hline & $\begin{array}{l}\text { BC Women's Hospital } \\
2 \text { - Clinical/Research } \\
\text { (Obstetrical anesthesia only) }\end{array}$ & 2009-2010 & $\begin{array}{l}\text { Dr. Roanne Preston, Head, Department of Anesthesia, BC Women's } \\
\text { Hospital, Room lQ72 - 4500 Oak St., Vancouver, BC V6H 3N1. Phone: } \\
\text { 604-875-2158; Fax: 604-875-2733; E-mail: rpreston@cw.bc.ca }\end{array}$ \\
\hline & $\begin{array}{l}\text { St. Paul's Hospital } \\
\text { l - Clinical/Research }\end{array}$ & 2009-2010 & $\begin{array}{l}\text { Dr. Randy Moore, Department of Anesthesia, St. Paul's Hospital, c/o } \\
\text { Operating Rooms, 1081 Burrard St., Vancouver, BC V6Z 1Y6. } \\
\text { Phone: 604-806-8337; Fax: 604-806-8487; E-mail: rmoore@ } \\
\text { providencehealth.bc.ca }\end{array}$ \\
\hline $\begin{array}{l}\text { University of Alberta } \\
\text { Edmonton, Alberta }\end{array}$ & $\begin{array}{l}\text { 1 - Liver Transplant and Trauma } \\
1 \text { - Cardiac Anesthesia } \\
\text { 1 - Cardiac Anesthesia } \\
\text { 1 - Pediatric Anesthesia } \\
\text { 1 - Regional Anesthesia } \\
\text { 1 - Regional Anesthesia } \\
\text { 1 - Regional Anesthesia } \\
\text { 1 - Regional Anesthesia }\end{array}$ & $\begin{array}{l}\text { July } 1,2008 \\
\text { July } 1,2008 \\
\text { October } 1,2009 \\
\text { July } 1,2008 \\
\text { January } 1,2008 \\
\text { October } 1,2008 \\
\text { July } 1,2009 \\
\text { August } 1,2009\end{array}$ & $\begin{array}{l}\text { The Chairman, Department of Anesthesiology and Pain Medicine, University } \\
\text { of Alberta Hospitals, 8-120 Clinical Sciences Building, Edmonton, Alberta } \\
\text { T6G 2G3. Phone: 780-407-8887. E-mail: chassist@ualberta.ca }\end{array}$ \\
\hline $\begin{array}{l}\text { Queen's University } \\
\text { Kingston, Ontario }\end{array}$ & 1-2 Clinical Anesthesia & $2009 / 2010$ & $\begin{array}{l}\text { Dr. David Mark, Fellowship Coordinator, Department of Anesthesiology, } \\
\text { Queen's University, Kingston General Hospital, 76 Stuart Street, Kingston, } \\
\text { Ontario K7L 2V7. Phone: 613-548-7827; Fax: 613-548-1375; } \\
\text { E-mail: asselstk@kgh.kari.net }\end{array}$ \\
\hline $\begin{array}{l}\text { Université de } \\
\text { Montréal, } \\
\text { Montréal, Québec }\end{array}$ & $\begin{array}{l}\text { 1 - Pediatrics/Obstetrics } \\
\text { (Clinical) } \\
1 \text { - Cardiovascular (Clinical/ } \\
\text { Research) } \\
\text { 1 - Transoesophageal } \\
\text { Echocardiography (Clinical) } \\
\text { 1 - Regional (Clinical) } \\
\text { 1 - Acute and/or Chronic Pain } \\
\text { (Clinical) } \\
\text { 1 - Neuroanesthesia (Clinical/ } \\
\text { Research) } \\
\text { 1 - Hepato-biliary (Clinical) } \\
\text { 1 - Thoracic (Clinical/ } \\
\text { including Lung } \\
\text { Transplantation) } \\
\text { 1 - Pharmacology } \\
\text { (Neuromuscular Blocking } \\
\text { Drugs) } \\
\text { 1 - Pharmacology (Pain) } \\
\text { 1 - Transfusion Medicine } \\
\text { (Clinical/Reseach) }\end{array}$ & $2009 / 2010$ & $\begin{array}{l}\text { Dr. Pierre Drolet, Chairman, Département d'anesthésiologie, C.P. 6128, } \\
\text { succursale A, Montréal, Québec H3C 3J7. Phone: 514-343-6466; } \\
\text { Fax: 514-343-6961; E-mail: pierre.drolet@umontreal.ca }\end{array}$ \\
\hline
\end{tabular}

General conditions

1. Qualification: FRCPC or eligibility for examination, except Vancouver General Hospital (completed minimum 5 years in Resident program) and University of Toronto Grant Dependent Positions (minimum 2 years in Resident program).

2. Remuneration, Medical Licencing Requirements, and other conditions: Contact individual departments.

3. Fellowships available for Canadian Citizens or Landed Immigrants.

4. Two-year program; possible one year overlap with base program of anesthesia. Required: FRCPC or eligibility for exams by end of program.

(List compiled June 2008 by Dr. Donald R. Miller, Editor-in-Chief, Canadian Journal of Anesthesia, Ottawa, Ontario). 